\title{
ON FRÉCHET DIFFERENTIABILITY OF LIPSCHITZIAN FUNCTIONS ON SPACES WITH GAUSSIAN MEASURES
}

\author{
V.I. BOGACHEV, E. PRIOLA, N.A. TOLMACHEV
}

\begin{abstract}
We construct two counter-examples related to Fréchet differentiability in infinite dimensions. The first one gives a convex Lipschitzian function on a Banach space such that its convolution with a given measure is Fréchet differentiable only on a measure zero set. The second one gives a Borel function on a space with a Gaussian measure such that it is Lipschitzian along the Cameron-Martin subspace, but is Fréchet differentiable along this subspace only on a measure zero set. This answers a long standing open question.
\end{abstract}

\section{AMS Subject Classification: 28C20, 49J50}

The problem of Fréchet differentiability of Lipschitzian functions has attracted a considerable attention in the last decades. One of the major achievements in this area is Preiss's theorem [1] according to which every Lipschitzian function on a Hilbert space is Fréchet differentiable on a dense set. However, this set may be small in many respects, in particular, it may have measure zero with respect to every nondegenerate Gaussian measure. The consideration of Gaussian measures on infinite dimensional spaces in relation with Fréchet differentiability brings new problems that are specifically infinite dimensional. Every Radon Gaussian measure $\gamma$ on a space $X$ (which is a Banach space or, more generally, a locally convex space) possesses the so called Cameron-Martin space $H$ (called also the reproducing kernel), which is a separable Hilbert space with some norm $|\cdot|_{H}$ and is compactly embedded into $X$. If $X$ is infinite dimensional, then $H$ is much smaller than $X$, although it may be dense in $X$. For many reasons, it is natural to consider functions on $X$ that are Fréchet differentiable along $H$. A function $f$ on $X$ is called Fréchet differentiable along $H$ (or Fréchet $H$-differentiable) at a point $x \in X$ if there is a vector $D_{H} f(x) \in H$ such that

$$
f(x+h)-f(x)-\left(D_{H} f(x), h\right)_{H}=o(h), \quad h \in H,
$$

where $\lim _{|h| \rightarrow 0}|h|_{H}^{-1}|O(h)|_{H}=0$. It turns out that this weaker property is much more flexible and that many natural functions are Fréchet differentiable along $H$ not even being continuous on $X$. For example, the convolution

$$
f * \gamma(x)=\int_{X} f(x+y) \gamma(d y)
$$

is infinitely Fréchet differentiable along $H$ for every function $f \in L^{2}(\gamma)$. If $X$ is a separable Banach space, then every Lipschitzian function $f$ on $X$ is $\gamma$-almost everywhere Gâteaux differentiable along $X$, which yields that $\gamma$-almost everywhere 
$f$ is Fréchet differentiable along $H$ (see Theorem 5.11.1 in [2]). In addition, if $H$ is dense in $X$, then, for any function $f$ that is Lipschitzian along the whole space $X$, the convolution $f * \gamma$ is everywhere Gâteaux differentiable along $X$ (since a Lipschitzian function differentiable along a dense subspace is Gâteaux differentiable; see, e.g., Lemma 1 in [3]).

Once we consider the differentiability along $H$, it is natural to introduce the Lipschitz condition also along $H$, i.e., the property that

$$
|f(x+h)-f(x)| \leq C|h|_{H}, \quad h \in H .
$$

If $f$ is a $\gamma$-measurable function and (1) holds for $\gamma$-almost every $x$, then $f$ is called $H$-Lipschitzian or Lipschitzian along $H$. In this case there is a version of $f$ that satisfies (1) for every $x \in X$; in addition, any $H$-Lipschitzian function is Gâteaux differentiable along $H$ at $\gamma$-almost every point in $X$ (see Theorem 5.11 .2 in [2]). We recall that $f$ is Gâteaux differentiable along $H$ at a point $x \in X$ if there is an element $D_{H} f(x) \in H$ such that for each $h \in H$ one has

$$
f(x+t h)-f(x)-t\left(D_{H} f(x), h\right)_{H}=r(h, t), \quad \lim _{t \rightarrow 0}\left|t^{-1} r(t, h)\right|_{H}=0 .
$$

These results lead to the following two natural questions:

(Q1) Can it happen that the convolution $f * \gamma$, where $f$ is a Lipschitzian function on a separable Hilbert space $X$ equipped with a nondegenerate Gaussian measure $\gamma$, has no Fréchet derivative along $X$ almost everywhere with respect to $\gamma$ ?

(Q2) Can it happen that a function $f$ on $X$ that is Lipschitzian along $H$ has no Fréchet derivative along $H$ almost everywhere with respect to $\gamma$ ?

The first question arises in relation to smoothing by the Ornstein-Uhlenbeck semigroup and other convolutions, which is a standard tool in stochastic analysis (see, e.g., [2], [3], and [4] and the references therein).

It was conjectured in [5] that an answer to Question (Q2) is affirmative (this question was mentioned also on p. 266 in [2]). Partial solutions have been obtained since then. In particular, an example of an $H$-valued mapping (in place of a real function) with the desired property is easy (see Example 5.11.4 in [2]), and an example in the case of a real function and some product-measure in place of $\gamma$ is constructed in [6]. The purpose of this paper is to construct two examples that confirm the expected positive answers to both questions. Thus, in spite of the fact that the convolution with a Gaussian measure $\gamma$ improves Gâteaux differentiability of a Lipschitzian function along the whole space and ensures smoothness along the densely embedded Cameron-Martin space of $\gamma$, it does not significantly improve Fréchet differentiability along $X$. Similarly, the Lipschitz condition along $H$ does not guarantee Fréchet differentiability along $H$ on a positive measure set.

Concerning Radon measures on topological spaces we refer to [7]. We recall that a Radon probability measure $\gamma$ on a locally convex space $X$ with the topological dual space $X^{*}$ is called a centered Gaussian measure if every continuous linear functional on $X$ is a centered Gaussian random variable on $(X, \gamma)$. If the distribution of any nonzero functional is not concentrated at a point, then $\gamma$ is called nondegenerate. 
The Cameron-Martin space $H$ of $\gamma$ is the set of all vectors $h \in X$ such that

$$
|h|_{H}:=\sup \left\{l(h): l \in X^{*},\|l\|_{L^{2}(\gamma)} \leq 1\right\}<\infty .
$$

It is known that there is an inner product $(\cdot, \cdot)_{H}$ on $H$ such that $|h|_{H}^{2}=(h, h)_{H}$ and $H$ is a separable Hilbert space with respect to this inner product. For more details on Gaussian measures, see [2]. In fact, for our purposes it suffices to consider the space $\mathbb{R}^{\infty}$, i.e., the countable power of the real line, and the standard Gaussian productmeasure $\gamma$ on $\mathbb{R}^{\infty}$, i.e., the countable power of the standard Gaussian measure on the real line. The Cameron-Martin space of this measure is the usual Hilbert space $l^{2}$ with its natural inner product. Similarly, one can consider the product $\mu$ of the sequence of Gaussian measures on the real line with densities

$$
p_{\sigma_{n}}(x)=\left(2 \pi \sigma_{n}\right)^{-1 / 2} \exp \left(-t^{2} /\left(2 \sigma_{n}\right)\right), \quad \sigma_{n}>0 .
$$

The Cameron-Martin space of this measures is the space of all sequences $h=\left(h_{n}\right)$ such that $\sum_{n=1}^{\infty} \sigma_{n}^{-1} h_{n}^{2}<\infty$. If $\sum_{n=1}^{\infty} \sigma_{n}<\infty$, then $\mu\left(l^{2}\right)=1$ and $\mu$ can be restricted to the Hilbert space $l^{2}$.

Our examples are based on the consideration of distance functions as in [8]. We recall that any $H$-Lipschitzian function belongs to all $L^{p}(\gamma)$ with $p<\infty$ (see Theorem 4.5.7 in [2]).

Note that the convolution $f * \mu$ is defined by the formula

$$
f * \mu(x)=\int_{X} f(x+y) \mu(d y)
$$

for any Radon measure $\mu$ and any function $f$ such that the functions $y \mapsto f(x+y)$ are integrable with respect to $\mu$.

Theorem 1. Let $X$ be an infinite dimensional separable Banach space and let $\mu$ be a Borel probability measure on $X$ such that the norm is $\mu$-integrable. There exists a convex Lipchitzian function $f$ on $X$ such that at $\mu$-almost every point $x \in X$ the convolution $f * \mu$ is not Fréchet differentiable along $X$.

Proof. We can find a balanced convex compact set $K$ such that $\mu(K)>0$ and $\mu\left(\cup_{n=1}^{\infty} n K\right)=1$ (see Theorem 7.12.4 in [7]). Set $A:=2 K$. Let us take a sequence of elements $v_{n} \in X$ such that $\left\|v_{n}\right\| \rightarrow 0$ and the distance between $A$ and $A+v_{n}$ is greater than $\left\|v_{n}\right\| / 8$. This is possible since, for every $n$, there is a vector $x_{n}$ with $\left\|x_{n}\right\|=1 / n$ which is separated from $2 A$ by a closed hyperplane of the form $\left\{x: l_{n}(x)=c_{n}\right\}, l_{n} \in X^{*}$, i.e., $l_{n}(a) \leq c_{n}$ if $a \in 2 A$ and $l_{n}\left(x_{n}\right)>c_{n}$. Set $Z_{n}:=\operatorname{Ker} l_{n}$ and take any element $z_{n} \in Z_{n}$ with $\left\|x_{n}-z_{n}\right\| \leq 2 \operatorname{dist}\left(x_{n}, Z_{n}\right)$, where $\operatorname{dist}(x, M):=$ $\inf \{\|x-m\|: m \in M\}$ for any set $M$. Then $v_{n}:=2\left(x_{n}-z_{n}\right)$ is a suitable vector. Indeed, we have

$$
\begin{aligned}
\operatorname{dist}\left(v_{n}, 2 A\right) & \geq \operatorname{dist}\left(v_{n}, x_{n}+Z_{n}\right)=\operatorname{dist}\left(x_{n}-2 z_{n}, Z_{n}\right) \\
& =\operatorname{dist}\left(x_{n}, Z_{n}\right) \geq \frac{1}{2}\left\|x_{n}-z_{n}\right\|=\frac{1}{4}\left\|v_{n}\right\| .
\end{aligned}
$$

Hence for any $a_{1}, a_{2} \in A$ we obtain $\left\|a_{1}-a_{2}-v_{n}\right\| \geq\left\|v_{n}\right\| / 4$. Note that $\left\|v_{n}\right\| \leq 4 / n$.

Let us consider the function

$$
f(x)=\operatorname{dist}(x, A)=\inf \{\|x-y\|: y \in A\},
$$


which is convex by the convexity of $A$ and Lipschitzian with constant 1 . Let us show that the convolution $f * \mu$ (which is defined since $f$ is Lipschitzian and the norm is $\mu$-integrable) is not Fréchet differentiable at the points of $K$. Clearly, $f * \mu$ is convex and Lipschitzian with constant 1. Suppose that the function $f * \mu$ is Fréchet differentiable at some point $x \in K$. Then, for any $\mu$-measurable set $E$, the function

$$
F_{E}(z)=\int_{E} f(z+y) \mu(d y)
$$

is Fréchet differentiable at $x$ too. This follows from the fact that $F_{E}$ and $F_{X \backslash E}$ are convex and continuous, and the sum of two convex continuous functions is Fréchet differentiable at $x$ if and only if each of them is Fréchet differentiable at $x$ (see [9], p. 86). Now we take $E=K$ and set $F:=F_{K}$. Since $x \in K$, one has $x+y \in A$ for all $y \in K$, hence $F(x)=0$. Therefore, since $x$ is a minimum point of $F$, we have $D F(x)=0$, where $D F(x)$ is the Fréchet derivative of $F$ at the point $x$. In order to obtain a contradiction, it suffices to show that $F(x+h)$ is not $o(|h|)$, i.e., there is a sequence of vectors $h_{n}$ with $\left\|h_{n}\right\| \rightarrow 0$ and $F\left(x+h_{n}\right) \geq c\left\|h_{n}\right\|$ with some $c>0$ independent of $n$. It would be enough to have an estimate

$$
f\left(x+y+h_{n}\right) \geq c_{1}\left\|h_{n}\right\|
$$

for each $y \in K$ with some constant $c_{1}>0$. Then we can take $c=c_{1} \mu(K)$. Let $h_{n}=$ $v_{n}$ be the vectors constructed above and let $B(a, r)$ denote the closed ball of radius $r$ in $X$ centered at $a$. Let $y \in K$. We observe that $B\left(x+y+v_{n},\left\|v_{n}\right\| / 8\right) \cap A=\emptyset$. Indeed, if $u \in A$, then $\left\|x+y+v_{n}-u\right\|>\left\|v_{n}\right\| / 8$ since $x+y \in A$. Therefore, one has the estimate $f\left(x+y+v_{n}\right) \geq\left\|v_{n}\right\| / 8$. So we can take $c_{1}=1 / 8$. Thus the set of points of Fréchet differentiability of $f * \mu$ does not meet the set $K$. Finally, replacing $K$ by $n K$ in the described construction, we obtain the corresponding function $f_{n}$ such that $f_{n}$ is Lipschitzian with constant 1 and convex and the set of Fréchet differentiability of the function $f_{n} * \mu$ does not meet $n K$. The function $f:=\sum_{n=1}^{\infty} 2^{-n} f_{n}$ is also convex and Lipschitzian with constant 1. As explained above, the function $f * \mu$ is not Fréchet differentiable at the union of the sets $n K$ (which is a set of full $\mu$ measure) because both functions $2^{-n} f_{n} * \mu$ and $\sum_{k \neq n} 2^{-k} f_{k} * \mu$ are Lipschitzian and convex.

We recall that in the case of a nondegenerate Gaussian measure $\mu$ on a Hilbert space $X$, for any convex Lipschitzian function $f$, the convex and Lipschitzian convolution $f * \mu$ is everywhere Gâteaux differentiable along $X$ and infinitely Fréchet differentiable along $H(\mu)$. In addition, this convolution is Fréchet differentiable along $X$ on a dense set.

Theorem 2. There is a Borel function $f$ on $\mathbb{R}^{\infty}$ that is Lipschitzian along $H$ with constant 1, but the set of points where $f$ is Fréchet differentiable along $H$ has $\gamma$ measure zero.

Moreover, such a function exists for every Radon centered Gaussian measure $\mu$ on a locally convex space $X$ such that the Cameron-Martin space $H=H(\mu)$ of $\mu$ is infinite dimensional. 
Proof. Let $\gamma_{n}$ be the standard Gaussian measure with density $\varrho_{n}$ in $\mathbb{R}^{n}$ and let $0<\varepsilon<1 / 2$. We show that there is a set $A_{n} \subset \mathbb{R}^{n}$ with the following properties: there exist a natural number $M_{n}$, points $a_{n, i}$ and positive numbers $r_{n, i}$ such that

$$
A_{n}=\bigcup_{i=1}^{M_{n}} B\left(a_{n, i}, r_{n, i}\right) \backslash B\left(a_{n, i}, \varepsilon r_{n, i}\right),
$$

where the closed balls $B\left(a_{n, i}, r_{n, i}\right)$ are pairwise disjoint, and $\gamma_{n}\left(A_{n}\right)>1-9 \varepsilon 2^{-n}$.

Indeed, we take a cube $Q$ with $\gamma_{n}(Q)>1-\varepsilon 4^{-n}$. Then we find $r \in\left(0,2^{-n}\right)$ such that

$$
\sup _{x \in B(a, r)} \varrho_{n}(x) \leq 2 \inf _{x \in B(a, r)} \varrho_{n}(x)
$$

for every ball $B(a, r) \subset Q$. Next we find finitely many pairwise disjoint balls $B\left(a_{n, i}, r_{n, i}\right) \subset Q, i=1, \ldots, M_{n}$, with $0<r_{n, i}<r$ such that the $\gamma_{n}$-measure of their union is greater than $1-\varepsilon 4^{-n}$. Let $\lambda_{n}$ be Lebesgue measure on $\mathbb{R}^{n}$. For every ball $B(a, \delta) \subset Q$ with $\delta<r$ we have

$$
\gamma_{n}(B(a, \varepsilon \delta)) \leq 2 \varepsilon^{n} \gamma_{n}(B(a, \delta)),
$$

which follows from (2) and the equality $\lambda_{n}(B(a, \varepsilon \delta))=\varepsilon^{n} \lambda_{n}(B(a, \delta))$. Therefore,

$$
\begin{aligned}
\gamma_{n}\left(\bigcup_{i=1}^{M_{n}} B\left(a_{n, i}, r_{n, i}\right) \backslash B\left(a_{n, i}, \varepsilon r_{n, i}\right)\right) & \geq\left(1-4 \varepsilon^{n}\right) \gamma_{n}\left(\bigcup_{i=1}^{M_{n}} B\left(a_{n, i}, r_{n, i}\right)\right) \\
>\left(1-4 \varepsilon^{n}\right)\left(1-\varepsilon 4^{-n}\right) & >1-\varepsilon 4^{-n}-4 \varepsilon^{n}>1-9 \varepsilon 2^{-n} .
\end{aligned}
$$

We represent $\mathbb{R}^{\infty}$ as $\mathbb{R}^{1} \times \mathbb{R}^{2} \times \mathbb{R}^{3} \times \cdots$ and the measure $\gamma$ as the product of the measures $\gamma_{n}$. Let $\varepsilon$ be fixed. For every natural number $n$ we take the set $A_{n} \subset \mathbb{R}^{n}$ constructed above and define a Borel set in $\mathbb{R}^{\infty}$ by

$$
A:=\prod_{n=1}^{\infty} A_{n} .
$$

Then we have

$$
\gamma(A) \geq \prod_{n=1}^{\infty}\left(1-9 \varepsilon 2^{-n}\right) \geq 1-9 \varepsilon .
$$

Finally, we define a Borel function $f$ on $\mathbb{R}^{\infty}$ by the formula

$$
f(x):=\operatorname{dist}_{H}(x, A):=\inf \left\{|x-y|_{H}: y \in A\right\}
$$

if there is at least one element $y \in A$ with $x-y \in H$. Otherwise we set $f(x)=0$. The fact that $f$ is Borel measurable follows from the equality

$$
\{x: f(x)<c\}=(A+c U) \cup(X \backslash(A+H)), \quad c>0,
$$

where $U$ is the open unit ball of $H$. The sets $A+c U$ and $A+H$ are Borel because $A+c U$ is the union of the sets $A+c V_{n}$, where $V_{n}$ is the closed ball of radius $1-1 / n$ in $H$, and these sets are compact in $X$. One has

$$
|f(x+h)-f(x)| \leq|h|_{H}, \quad h \in H,
$$


i.e., this function is Lipschitzian along $H$. Let us show that $f$ is not Fréchet differentiable along $H$ at the points of the set $A$. Indeed, suppose that $a \in A$ is such that $f$ is Fréchet differentiable along $H$ at $a$. Since $f(a)=0$ and $f \geq 0$, we conclude that $D_{H} f(a)=0$. This will yield a contradiction if we show that, for any $\delta>0$, there is a vector $h \in H$ with $|h|_{H}<\delta$ such that

$$
\left\{x:|a+h-x|_{H} \leq \varepsilon|h|_{H}\right\} \cap A=\emptyset .
$$

Indeed, this relationship gives the inequality $f(a+h) \geq \varepsilon|h|_{H}$, which contradicts the fact that the Fréchet derivative of $f$ along $H$ vanishes at the point $a$. We have $a=\left(a_{n}\right)$, where $a_{n} \in \mathbb{R}^{n}$ for every $n$. Let us pick $n$ such that $2^{-n}<\delta$. Then $a_{n} \in B\left(a_{n, i}, r_{n, i}\right) \backslash B\left(a_{n, i}, \varepsilon r_{n, i}\right)$ for some $i \leq M_{n}$. It remains to take the vector $h$ whose $n$th component in our representation of $\mathbb{R}^{\infty}$ is $a_{n, i}-a_{n}$ and all other components are zero. Then we obtain $|h|_{H} \leq r_{n, i}<2^{-n}<\delta$. In addition, the ball of radius $\varepsilon|h|_{H}$ in the metric of $H$ centered at $a+h$ does not meet $A$ since otherwise the ball in $\mathbb{R}^{n}$ of radius $\varepsilon r_{n, i}$ centered at $a_{n, i}$ would meet the set $A_{n}$, which is impossible by our construction.

The previous step gives a function $f$ that is Lipschitzian with constant 1 , but is not Fréchet differentiable at the points of the set $A$ with $\gamma(A) \geq 1-8 \varepsilon$. Now we construct such a set for every $\varepsilon=1 / k, k \in \mathbb{N}$, and denote the corresponding function by $f_{k}$. Let us represent $X=\mathbb{R}^{\infty}$ as the product of countably many copies $X_{k}$ of the space $\mathbb{R}^{\infty}$, equip it with the standard Gaussian product-measure $\gamma$ (which coincides with the countable product of the standard Gaussian product-measures $\gamma^{(k)}$ on the factors $X_{k}$ ), and denote by $\pi_{k}$ the projection operator to the $k$ th factor. The Cameron-Martin space $H$ of $\gamma$ coincides with $l^{2}$ and equals the Hilbert sum of the Cameron-Martin spaces $H_{k}$ of the measures $\gamma^{(k)}$. The function

$$
f(x):=\sum_{k=1}^{\infty} 2^{-k} f_{k}\left(\pi_{k}(x)\right)
$$

is Lipschitzian along $H$ with constant 1 . This function is not Fréchet differentiable along $H$ almost everywhere with respect to the measure $\gamma$. Indeed, for every $k$, let us set $E_{k}:=A_{k} \times \prod_{n \neq k} X_{n}$. We can write $f=2^{-k} f_{k}+g_{k}$, where $g_{k}:=\sum_{n \neq k} 2^{-n} f_{n}$. The function $g_{k}$ does not depend on $\pi_{k}(x)$. Since $f_{k}$ is not Fréchet differentiable along $H_{k}$ at the points of the set $A_{k}$, the function $f$ is not Fréchet differentiable along $H_{k}$ (hence also along $H$ ) at the points of the set $E_{k}$. It remains to observe that $\gamma\left(E_{k}\right)=\gamma^{(k)}\left(A_{k}\right) \geq 1-9 k^{-1}$.

The case of a general locally convex space $X$ equipped with a centered Radon Gaussian measure $\mu$ whose Cameron-Martin space $H(\mu)$ is infinite dimensional reduces to the considered case. Indeed, according to Theorem 3.4.4 in [2], there exists an injective Borel measurable linear mapping $T$ defined on a Borel linear subspace $E \subset \mathbb{R}^{\infty}$ with $\gamma(E)=1$ such that $E_{0}:=T(E)$ is a Borel Souslin subspace in $X$ with $\mu\left(E_{0}\right)=1, \mu$ coincides with the image of $\gamma$ under $T$, and $T$ is an isometry between $l^{2}$ and $H(\mu)$. The mapping $S:=T^{-1}: E_{0} \rightarrow E$ is Borel measurable (see Theorem 6.8.6 in [7]). Having constructed our function $f$ on $\mathbb{R}^{\infty}$, we obtain a Borel measurable function $f_{0}=f \circ S$ on $E_{0}$ with the required properties. Outside $E_{0}$ we set $f_{0}(x)=0$. 
This theorem gives a partial answer to Question (iii) on p. 266 in [2] concerning the set of points of Fréchet $H$-differentiability of the function $\operatorname{dist}_{H}(x, B)$.

We do not know whether there is a Lipschitzian function $f$ on a separable Hilbert space $X$ such that its convolution $f * \mu$ with some nondegenerate Gaussian measure $\mu$ on $X$ is Fréchet differentiable only on a set that has measure zero with respect to all nondegenerate Gaussian measures on $X$, i.e., is a so called Gaussian null set. One might also wonder whether a Lipschitzian function $f$ may have such a property for every Gaussian measure $\mu$ (i.e., not just for some $\mu$ ). It is known (see [10] and [9, p. 157]) that on $X=l^{2}$ there is a convex function which is Lipschitzian along the whole space $X$ and whose Fréchet differentiability set (along the whole space $X$ ) is a Gaussian null set. A similar question can be posed in relation to Question (Q2) above. Namely, let $f$ be a continuous function on a separable Hilbert space (or on $\mathbb{R}^{\infty}$ ) such that it is Lipschitzian along the Cameron-Martin space $H$ of some nondegenerate Gaussian measure $\gamma$. Can it happen that the set of points where $f$ is Fréchet differentiable along $H$ is a Gaussian null set? Finally, it would be interesting to know whether in Theorem 2 one can find a function $f$ with the additional property that it is convex along $H$. In particular, it is not clear whether $f$ can be found in the form $\operatorname{dist}_{H}(x, A)$ for some Borel set $A$ that is convex along $H$.

This work was supported by the RFBR project 04-01-00748, the INTAS project 99-559, the SFB-701 at the University of Bielefeld, the Russian-Japanese Grant 05-01-02941-JF, the DFG Grant 436 RUS 113/343/0(R), and the Italian National Project MURST "Equazioni di Kolmogorov".

\section{REFERENCES}

[1] Preiss D., Differentiability of Lipschitz functions on Banach spaces. J. Funct. Anal. 1990. V. 91. P. $312-345$.

[2] Bogachev V.I. Gaussian measures. Amer. Math. Soc., Providence, Rhode Island, 1998.

[3] Bogachev V.I., Smooth measures, the Malliavin calculus and approximation in infinite dimensional spaces. Acta Univ. Carol., Math. et Phys. 1990. V. 31, no. 2. P. 9-23.

[4] Priola E., Uniform approximation of uniformly continuous and bounded functions on Banach spaces. Dynamic Systems Appl. 2000. V. 9, no. 2. P. 181-197.

[5] Enchev O., Stroock D., Rademacher's theorem for Wiener functionals. Ann. Probab. 1993. V. 21, no. 1. P. 25-33.

[6] Bogachev V.I., Mayer-Wolf E., Some remarks on Rademacher's theorem in infinite dimensions. Potential Anal. 1996. V. 5, no. 1. P. 23-30.

[7] Bogachev V.I. Measure theory. V. 1,2. Springer, Berlin - Heidelberg, 2007.

[8] Preiss D., Zajiček L., Fréchet differentiation of convex functions in a Banach space with a separable dual. Proc. Amer. Math. Soc. 1984. V. 91. P. 202-204.

[9] Benyamini Y., Lindenstrauss J. Geometric nonlinear functional analysis. Amer. Math. Soc., Providence, Rhode Island, 2000.

[10] Matoušek J., Matouškovà E., A highly non-smooth norm on Hilbert space. Israel J. Math. 1999. V. 112. P. 1-27.

V.B., N.T.: Department of Mechanics and Mathematics, Moscow State Lomonosov University, 119992 Moscow, Russia

E.P.: Department of Mathematics, University of Torino, Torino 10123, Italy 